\title{
The Aurora-A inhibitor MLN8237 affects multiple mitotic processes and induces dose-dependent mitotic abnormalities and aneuploidy
}

\author{
Italia Anna Asteriti', Erica Di Cesare ${ }^{1}$, Fabiola De Mattia ${ }^{1}$, Volker Hilsenstein ${ }^{2}$, \\ Beate Neumann ${ }^{2}$, Enrico Cundari ${ }^{1}$, Patrizia Lavia ${ }^{1}$ and Giulia Guarguaglini ${ }^{1}$ \\ ${ }^{1}$ Institute of Biology, Molecular Medicine and Nanobiotechnology (formerly Institute of Molecular Biology and Pathology), \\ CNR National Research Council, c/o Department of Biology and Biotechnology, Sapienza University of Rome, Rome, Italy \\ ${ }^{2}$ Advanced Light Microscopy Facility, EMBL, Heidelberg, Germany \\ Correspondence to: Giulia Guarguaglini, email: giulia.guarguaglini@uniroma 1.it
}

Keywords: Aurora kinases, MLN8237, mitotic spindle, aneuploidy, time-lapse microscopy

Received: March 7, 2014

Accepted: July 8, 2014

Published: July 9, 2014

This is an open-access article distributed under the terms of the Creative Commons Attribution License, which permits unrestricted use, distribution, and reproduction in any medium, provided the original author and source are credited.

\section{ABSTRACT}

Inhibition of Aurora kinase activity by small molecules is being actively investigated as a potential anti-cancer strategy. A successful therapeutic use of Aurora inhibitors relies on a comprehensive understanding of the effects of inactivating Aurora kinases on cell division, a challenging aim given the pleiotropic roles of those kinases during mitosis. Here we have used the Aurora-A inhibitor MLN8237, currently under phase-I/III clinical trials, in dose-response assays in U2OS human cancer cells synchronously proceeding towards mitosis. By following the behaviour and fate of single Aurora-inhibited cells in mitosis by live microscopy, we show that MLN8237 treatment affects multiple processes that are differentially sensitive to the loss of Aurora-A function. A role of Aurora-A in controlling the orientation of cell division emerges. MLN8237 treatment, even in high doses, fails to induce efficient elimination of dividing cells, or of their progeny, while inducing significant aneuploidy in daughter cells. The results of single-cell analyses show a complex cellular response to MLN8237 and evidence that its effects are strongly dose-dependent: these issues deserve consideration in the light of the design of strategies to kill cancer cells via inhibition of Aurora kinases.

\section{INTRODUCTION}

The Aurora-A kinase is a major regulator of cell division and operates in distinct processes required for spindle assembly: in human cells it regulates separation and maturation of centrosomes at mitotic entry, mitotic microtubule (MT) nucleation [1-3] and the integrity of spindle poles $[2,4,5]$. Recent data also indicate a role of Aurora-A in central spindle assembly at telophase [6, 7]. The highly homologous Aurora-B kinase also operates in control of the fidelity of chromosome segregation, by regulating chromosome condensation, correction of improper attachments between MTs and kinetochores, spindle checkpoint function, cytokinesis and abscission [8].

As other mitotic regulators, Aurora kinases are often abnormally expressed in tumor cells and are being investigated as targets of anti-mitotic compounds for cancer therapy $[9,10]$. Many efforts have converged in the last years to develop Aurora inhibitors: molecules acting as ATP-competitors have been identified and some of them are currently in clinical trials [11]. Only a few of those molecules discriminate Aurora-A vs Aurora-B and may thus prove useful both in clinical studies for comparing the efficacy of anti-tumor responses and for dissecting the functions of Aurora kinases in mammalian cells.

MLN8237 (Alisertib) is a second generation Aurora inhibitor currently undergoing Phase-I/III clinical trials [11-16; www.clinicaltrials.gov]. Thus far, it is one of the molecules displaying highest specificity for Aurora-A over Aurora-B (300-fold in in vitro assays and 200-fold in HCT116 colorectal carcinoma cells [17]). Most pre- 
clinical studies based on whole cell population analyses in tumor cell lines showed cell growth inhibition, accumulation of polyploid cells over time, as well as induction of cell death [17-19]. Anti-tumor activity was also demonstrated in xenograft mouse models [17, 20, 21].

Available data on MLN8237-treated cells were mostly obtained from asynchronous cultures analyzed in bulk populations. This approach reveals the predominant cellular behaviour after long exposure to Aurora-A inhibition ( 24 to 96 hours) but can miss out transient phenomena and so mask the unfolding of relevant processes. In addition, inhibition of as pleiotropic a kinase as is Aurora-A, yields multiple phenotypes over time, making it difficult to dissect distinct functional roles within a bulk population. Microscopy-based single cell analyses are proving of critical importance to visualize the array of possible cell responses to anti-mitotic drugs [22]. Here we have coupled high resolution microscopy and high-throughput analysis of single cells treated with increasing doses of Aurora-A inhibitor to investigate the possible fates of cells with inactive Aurora-A.

A protocol was set up for treating pre-synchronized cultures when they reach G2 and analyze progression through G2 and mitosis as soon as Aurora-A inhibition is achieved. Because MLN8237 induces spindle pole abnormalities [23], we assessed the occurrence of chromosome mis-segregation events and aneuploidy induction, which would represent undesirable effects of the treatment in anti-cancer therapy. Our results highlight a partial specificity of MLN8237 in the U2OS cell line, with multiple cellular responses in a dose-dependent manner. The single cell analysis enabled us to depict a fraction of cells with defective spindle orientation, a defect that was not appreciated in previous studies of Aurora-A inhibition in human cells. In addition, we find that low and high MLN8237 concentrations yield mild and massive aneuploidy, respectively, representing a tumor-inducing or a tumor-suppressing condition [24]. Collectively, these results draw attention to the variability and the nature of cellular responses to the loss of Aurora kinase function, which may represent potential caveats deserving consideration when designing and interpreting clinical trials.

\section{RESULTS}

\section{MLN8237 displays dose-dependent target selectivity on Aurora kinases}

Prior to analyzing mitotic division in cells with inhibited Aurora-A, we sought to precisely define the specificity of MLN8237 inhibition in dose-response assays. We used the U2OS osteosarcoma cell line for its ease of cytological analysis, which renders it especially suitable for high-resolution single-cell microscopy analysis, and employed in our previous studies of RNA interference-mediated Aurora-A inactivation [4, 5, 23].

We set up a protocol by pre-synchronizing U2OS cells at the G1/S transition by thymidine treatment, then releasing from arrest into G2 and mitosis (Figure 1A). MLN8237 was added 6 hours after thymidine release (late S-phase/early G2) and cells were harvested after further 4 hours. Aurora-A activity was measured at the single cell level by anti-Aur-A-phospho-Thr288 immunofluorescence (IF) staining in dose-response assays (Figure 1B, left panels). Aurora-A auto-phosphorylation was significantly inhibited at concentrations ranging from $5 \mathrm{nM}$ to $250 \mathrm{nM}$. With concentrations higher than $20 \mathrm{nM}$ the residual signal at spindle poles was below $15 \%$ compared to controls. In Western blot analysis, no phospho-Thr288-Aurora-A was detectable in mitotic extracts from cultures treated with 20 and 50 nM MLN8237 for 4 hours, while some residual amount was present after 1 hour (Figure 1C).

Previous reports indicated that MLN8237 above $100 \mathrm{nM}$ also inhibits Aurora-B activity in other cell lines $[19,25-27]$. We therefore assessed the specificity of MLN8237 by measuring Aurora-B activity using anti-AurB-phospho-Thr232 antibody (Figure 1B, right panels). Surprisingly, we noticed that Aurora-B activity is already significantly compromised by $50 \mathrm{nM}$ MLN8237; that was not evident when using anti-phospho-Histone-H3 (Ser10) as a reporter of Aurora-B activity (Supplementary Figure $\mathrm{S} 1$ ), possibly reflecting kinase redundancy or delay in detecting modulation of phosphorylation of downstream targets $v s$ auto-phosphorylation.

Our single-cell analysis in U2OS cultures delimits therefore a narrow MLN8237 concentration window (20$50 \mathrm{nM}$ ) yielding effective and specific Aurora-A inhibition.

\section{MLN8237 delays mitotic entry and prolongs mitotic duration in a dose-dependent manner}

We investigated the influence of MLN8237 on mitotic entry: after 4 hours of treatment, we found a significantly lower percentage of mitotic cells in MLN8237-treated cultures compared to controls (Figure $2 \mathrm{~A}$ ). This effect is dose-dependent, appearing at $\geq 20 \mathrm{nM}$, and is stronger at $250 \mathrm{nM} \mathrm{MLN8237.} \mathrm{To} \mathrm{clarify} \mathrm{whether}$ cells were arrested in the G2 phase or rather delayed in progression through the $\mathrm{G} 2 / \mathrm{M}$ transition, we recorded cultures in time-lapse experiments from the treatment start up to 16 hours later (Figure 2B). The peak of interphases entering mitosis in control cultures (DMSO) was between 4 and 8 hours from the treatment start (Figure 2B) and was not significantly affected by partial Aurora-A inhibition (5-10 nM MLN8237). Entry into mitosis was instead delayed above $20 \mathrm{nM}$ MLN8237. In the 16 hours of time-lapse recording, $70-80 \%$ of the interphase cells entered mitosis in all treated cultures (about $60 \%$ with 
$250 \mathrm{nM}$ MLN8237): thus, the majority of cells exposed to MLN8237 are delayed in G2, yet mitotic onset is not prevented.

Extending the time-lapse recording to 30 hours indicated that MLN8237 prolonged the duration of mitosis in a dose-dependent manner (about 300 minutes with 50 $\mathrm{nM}$, compared to about 80 minutes in control cells; Figure 3). Importantly, although slowed down, cells eventually exited mitosis. Together, the results indicate that both the G2-to-mitosis transition and the overall duration of the mitotic process are strongly dependent on Aurora-A.

\section{Inhibition of Aurora kinases yields impaired MT nucleation, disorganized spindles and multipolar or failed cell division}

We next analyzed spindle structure in cells that entered mitosis with different degrees of Aurora-A and Aurora-B inhibition.

With the highest MLN8237 concentration (250 $\mathrm{nM}$ ) a strong impairment of MT nucleation was evident, with $70 \%$ of prometaphases displaying no MTs (Figure 4); this was associated with a prolonged prometaphase duration in time-lapse recording experiments, yielding an accumulation of prometaphase figures over all mitoses in fixed samples (Supplementary Figure S2). The MT nucleation defect was strongly dose-dependent and appeared in a relevant fraction of mitotic cells treated with 50 nM MLN8237 or above.

In cells in which MT nucleation was not visibly affected, spindles were highly disorganized (affecting 30\% to $60 \%$ of all prometaphases with $10 \mathrm{nM}$ or above; Figure $4)$; a fraction of these prometaphases displayed spindles with multiple poles, consistent with previous observations $[17,23]$. A non-significant fraction of monopolar spindles was present at 10-20 nM MLN8237.

The influence of these defects on the global execution of mitosis was examined in depth in timelapse movies of MLN8237-treated cells. Figure 5, first row, and Supplementary Movie S1 show examples of normal mitosis. We observed that a significant fraction of cells with inhibited Aurora-A (about 20\% with 50 $\mathrm{nM}$ MLN8237, and a smaller fraction with $250 \mathrm{nM}$ ) underwent multipolar ana-telophase (Figure 5, second row; Supplementary Movie S2). Multipolar mitoses took a longer time to reach the stage of chromosome segregation (about 125 minutes average, compared to 35 minutes in control cells). In some cases two of the multiple groups of segregating chromosomes eventually re-joined,
Figure 1: Dose-dependent inhibition of Aurora-A and Aurora-B by MLN8237. A. Protocol for MLN8237 treatment in cells progressing towards mitosis after thymidine (Thym) arrest and release. B. Quantification of IF signals for active pThr288-Aurora-A (left, mean intensity at poles) or active pThr232-Aurora-B (right, sum intensity at chromosomes) in control (DMSO) or MLN8237-treated prometaphases is shown in the box-plots (center lines show the medians; box limits indicate the 25th and 75th percentiles as determined by R software; whiskers extend 1.5 times the interquartile range from the 25th and 75th percentiles, outliers are represented by dots). Fluorescence intensity is shown in arbitrary units (a.u.). **: $\mathrm{p}<0.0001$, unpaired $\mathrm{t}$ test or Mann-Whitney test. $\mathrm{n}=90$ spindle poles ( $\mathrm{p}$-Aurora-A) or 50 prometaphases ( $\mathrm{p}$-Aurora-B) from 3 experiments. Representative IF images are shown. Scale bars: $10 \mu \mathrm{m}$. C. p-Aurora-A (active) levels decrease in mitotic extracts (shake-off) from MLN8237-treated (1 or 4 hours before harvesting) compared to DMSO- or nocodazole (NOC)-treated (4 hours) U2OS cultures. Total Aurora-A levels are also shown; actin is used as loading control. p-Aurora-B was not assessed in Western blot due to the lack of a suitable antibody for this application.

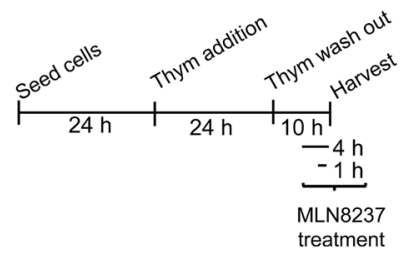

B.
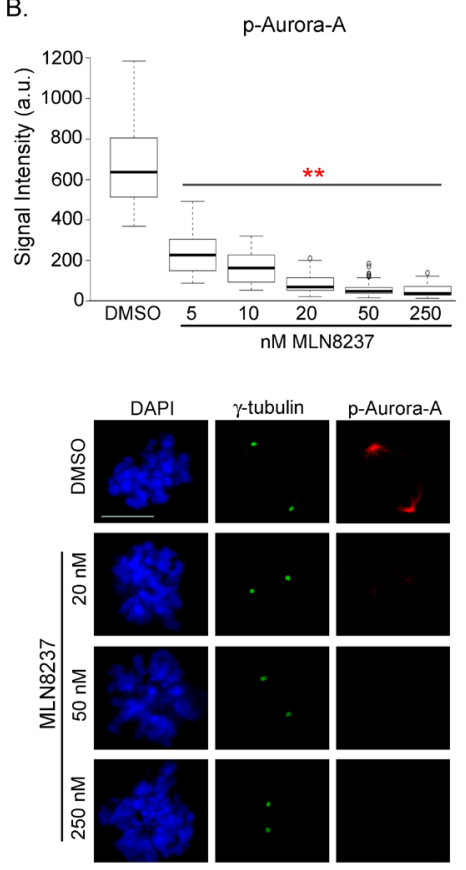

C.
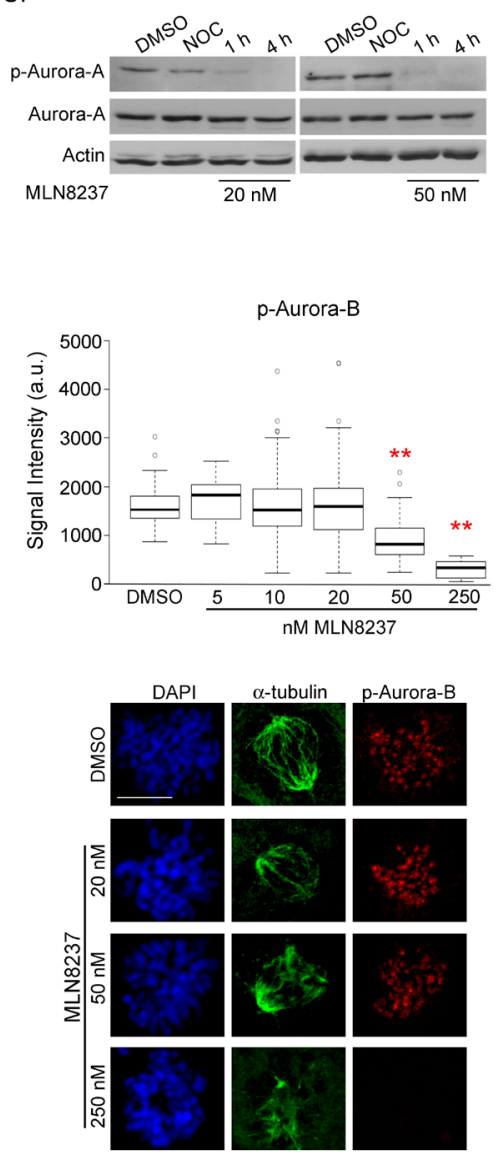

p-Aurora-B
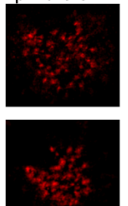
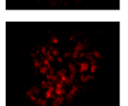

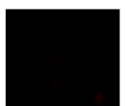


originating two asymmetric daughter cells (Figure 5, third row). Lower MLN8237 doses (10-20 nM), which yielded disorganized spindles in fixed prometaphases (Figure 4), do not yield multipolar divisions, suggesting that in those cultures a bipolar or pseudo-bipolar spindle is eventually assembled before anaphase.

In cultures treated with high MLN8237 doses we observed prolonged prometaphase (average duration 150-200 minutes) followed by a complete lack of chromosome segregation and cell division (Figure 5, fourth row): cells eventually re-adhered to form a single large or multinucleated interphase, often preceded by repeated "blebbing" movements (Figure 5, fifth row; Supplementary Movie S3). The "lack of division" phenotype appeared in a small fraction of mitoses treated with $50 \mathrm{nM}$ MLN8237 and became predominant (about $90 \%$ of mitoses) with $250 \mathrm{nM}$. Similar phenotypes were previously observed in other human cell lines treated with high MLN8237 concentrations [26, 27]. Since the

A.

\begin{tabular}{|c|c|c|c|c|}
\hline treatment & MI & $\boldsymbol{p}$ & $\mathbf{n}$ & $\exp$ \\
\hline DMSO & 7.35 & - & 2400 & 4 \\
\hline MLN8237 5 nM & 6.89 & n.s. & 1800 & 3 \\
\hline MLN8237 10 nM & 5.83 & n.s. & 1800 & 3 \\
\hline MLN8237 20 nM & 4.50 & $p<<0.001$ & 2400 & 4 \\
\hline MLN8237 50 nM & 3.87 & $p<<0.001$ & 2400 & 4 \\
\hline MLN8237 250 nM & 2.00 & $p<<0.001$ & 1800 & 3 \\
\hline
\end{tabular}

B.
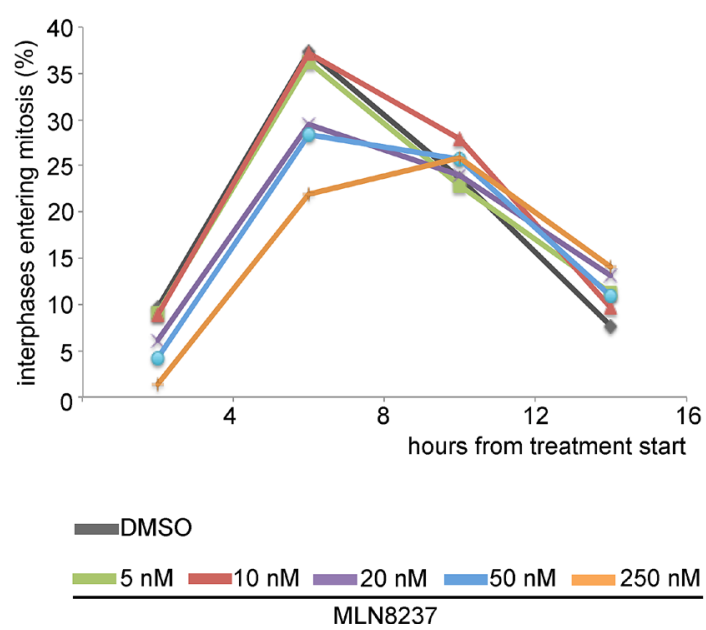

Figure 2: MLN8237 delays entry into mitosis. A. Mitotic index (MI) from control (DMSO) and MLN8237-treated cultures (protocol as in Figure 1A), as assessed by DAPI (DNA) and alpha-tubulin (spindle) staining. $p$ values $(\chi 2$ test) relative to control cultures, number of scored cells (n) and of independent experiments (exp) are indicated. B. Control (DMSO) and MLN8237-treated cultures were recorded by time-lapse imaging from the treatment start for the following 16 hours. The graph shows the percentage of interphases entering mitosis during the recording period; results are grouped in 4-hours intervals. 250 recorded interphases in 3 experiments for each condition. activity assays in Figure 1 indicate dual inhibition of both Aurora-A and -B under high doses, it was important to establish the contribution of each individual kinase to the no-division phenotype appearing above $50 \mathrm{nM}$. Smallscale time-lapse recording of cell cultures subjected to individual (Aurora-A or Aurora-B) or combined (AuroraA+Aurora-B) RNA interference were set up to clarify this issue (Supplementary Figure S3): the no-division phenotype was not recorded in mitoses with selective inactivation of Aurora-A alone, yet appeared in a small fraction of Aurora-B defective cells $(15,4 \%)$ and was amplified by the concomitant inactivation of both kinases $(41,9 \%)$.
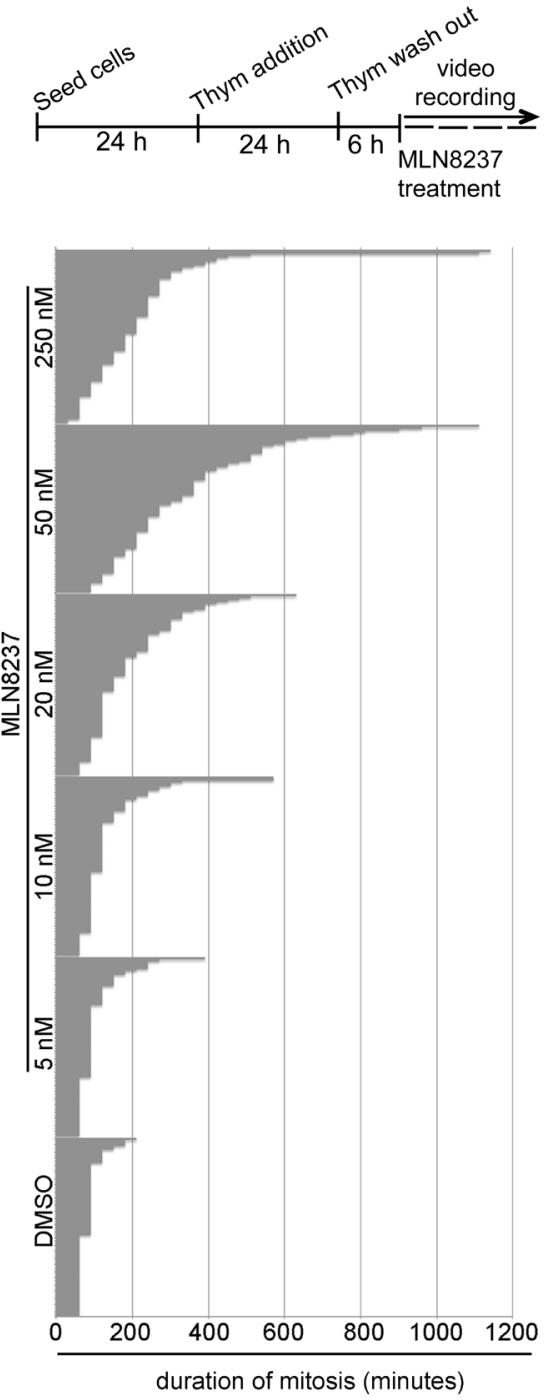

Figure 3: Dose-dependent lengthening of mitosis in MLN8237-treated cultures. The protocol for time-lapse recording of MLN8237-treated cultures progressing towards mitosis from thymidine (Thym) arrest and release is depicted on top. Phase-contrast microscopy images were acquired with a $10 \mathrm{x}$ objective every 30 minutes. Duration of mitosis is calculated from round-up to visualization of 2 distinct daughter cells; each bar represents a mitotic cell. At least 90 cells per condition are displayed from 3 experiments. 
In summary, these results show that spindle organization is the most sensitive process affected by MLN8237 and is readily altered by even a partial reduction of Aurora-A activity, associated with unbalanced chromosome segregation. Stronger inhibition of the kinase induces impairment in MT nucleation, associated with prolonged prometaphase duration. The highest MLN8237 dose leads to a complete failure of cell division, largely ascribable to the concomitant inhibition of Aurora-B.

\section{Aurora-A inactivation induces defects in the orientation of cell division}

The time-lapse recording experiments also revealed that a fraction of cells did not divide parallel to the growing surface: that was already evident under conditions of partial Aurora-A inhibiton, occurring in about $15 \%$ of mitoses in U2OS cultures treated with 5,10 or $20 \mathrm{nM}$ MLN8237 (Figure 6A-B and Supplementary Movie S4). These cells took a longer time to reach the stage of chromosome segregation

Figure 4: Spindle defects in MLN8237treated mitoses. Cultures harvested 4 hours after MLN8237 treatment (protocol as in Figure 1A) were stained for DNA and alphatubulin. Histograms represent the percentage of prometaphases displaying normal or defective spindles (IF panels on top). 250300 counted cells per condition from 3 experiments; s.d. are shown. Scale bar: $10 \mu \mathrm{m}$.

Figure 5: Time-lapse analysis of MLN8237-treated mitoses reveals multipolar and "no division" phenotypes. Cultures treated as in the protocol in Figure 3 were recorded by timelapse from treatment start for the following 24 hours. DIC images were acquired with a 40x objective every 5 minutes; representative single photograms are shown; time from round-up is indicated. First row: normal mitosis; second and third rows: multipolar mitoses ( $\mathrm{a}, \mathrm{b}$ and $\mathrm{c}$ indicate daughter cells); fourth and fifth rows: mitoses passing directly from prometaphase to defective interphase, with (lower) or without (upper) a "blebbing" phase. Defects are quantified (\%) in the table below; number of recorded mitoses (n) and independent experiments per condition are indicated. *: $0.01<\mathrm{p}<0.02$; **: $\mathrm{p}<0.001, \chi 2$ test. Scale bar: $10 \mu \mathrm{m}$. from mitotic round-up (on average 65 minutes with 5-10 $\mathrm{nM}$ and 110 with $20 \mathrm{nM}$ MLN8237) compared to control cells (35 minutes). The time from the onset of chromosome segregation to re-formation of daughter interphase cells was instead unaltered, indicating that the process of chromosome segregation per se was not disrupted. However, we often recorded a delay between re-adhesion of the lower and the upper cell (see the example in Figure 6A and Supplementary Movie S4). No significant induction of mis-oriented division was observed above $50 \mathrm{nM}$ MLN8237 (Figure 6B). In the case
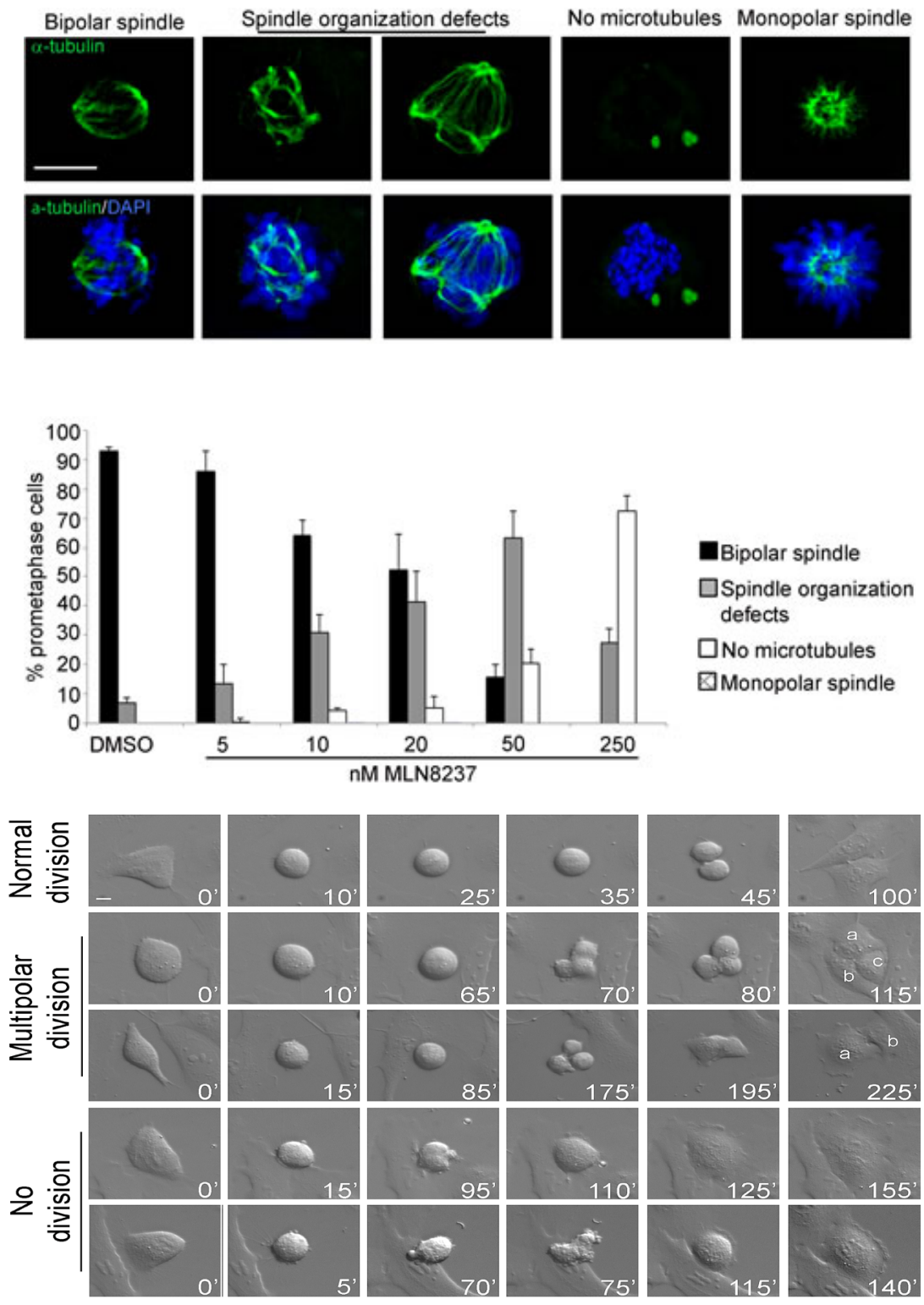

\begin{tabular}{c|c|c|c|c} 
treatment & multipolar (\%) & no division (\%) & $\mathbf{n}$ & exp \\
\hline DMSO & 1.60 & 0 & 187 & 3 \\
\hline MLN82375 nM & 3.26 & 1.63 & 184 & 3 \\
\hline MLN823710 nM & 2.81 & 2.25 & 178 & 4 \\
\hline MLN8237 20 nM & 4.88 & 1.22 & 84 & 3 \\
\hline MLN8237 50 nM & $21.54^{* *}$ & $8.46^{* *}$ & 130 & 3 \\
\hline MLN8237 250 nM & $7^{*} 23^{*}$ & $90.34^{* *}$ & 83 & 3
\end{tabular}


of $250 \mathrm{nM}$ MLN8237 treatment, this is consistent with the predominance of the no-division phenotype (see Figure 5). The absence of mis-oriented divisions with $50 \mathrm{nM}$, which induced highly disorganized spindles and/or defective MT nucleation (see Figure 4), suggests that MTs are required

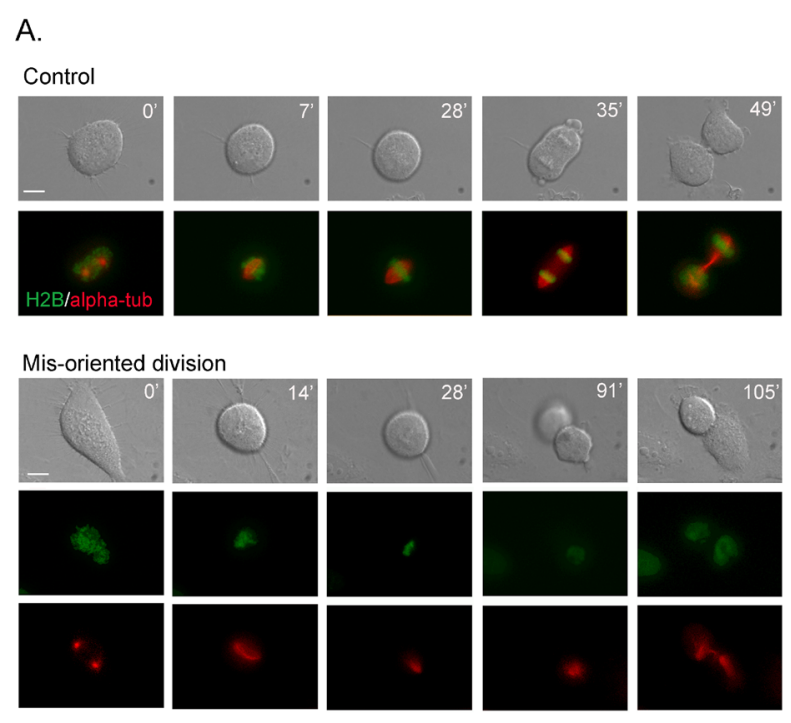

B.

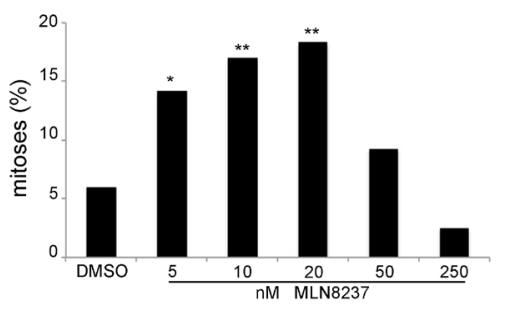

C.
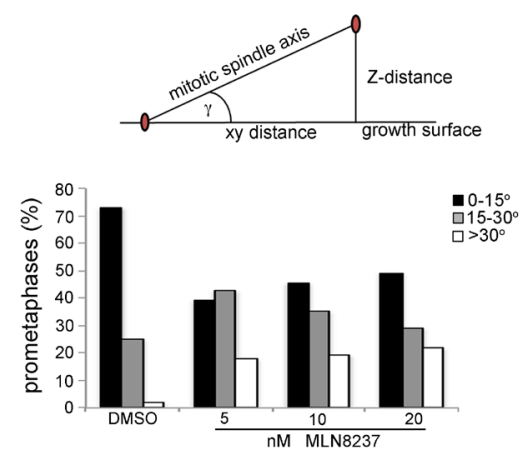

Figure 6: Aurora-A inhibition induces mis-oriented cell division. A. An example of normally dividing cell is shown on top. Mis-oriented division in the presence of MLN8237 in U2OS cells with fluorescently labeled H2B (green) and alpha-tubulin (red) is shown below. Minutes from round-up are indicated. Scale bars: $10 \mu \mathrm{m}$. B. Quantification (\%) of mis-oriented cell division recorded from MLN8237 treatment start for the following 20 hours (80-200 mitoses per condition from 3-4 independent experiments). $*: 0.01<\mathrm{p}<0.02 ; * *: \mathrm{p}<0.01, \chi 2$ test. C. The angle between the growth surface and the centrosome-centrosome axis in MLN8237-treated mitoses is calculated as schematized on top (centrosomes are in red; see Methods for details); histograms represent the distribution of prometaphases in 3 classes (50-60 cells from 3 independent experiments). and that spindle mis-orientation drives the abnormally oriented divisions.

To gain better resolution we used an U2OS cell line derivative stably expressing fluorescently labelled alpha-tubulin and histone $\mathrm{H} 2 \mathrm{~B}$ in video recoding assays (Supplementary Figure S4). MLN8237-treated mitoses that did not divide parallel to the culture dish displayed spindle rotation during prometaphase, such that often only one of the two poles was visible (Figure 6A, lower panels). To define the spindle orientation axis, we analyzed fixed samples and measured the angle formed between the centrosome-centrosome axis and the growing surface (Figure 6C). This analysis was performed in

A.

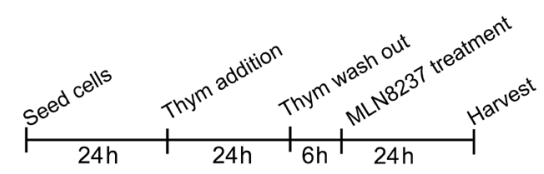

B.
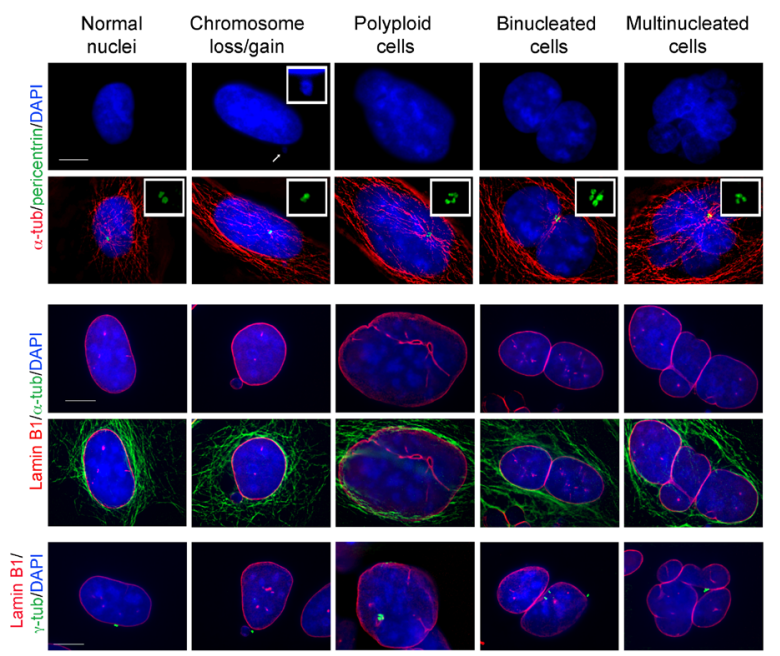

C. 100

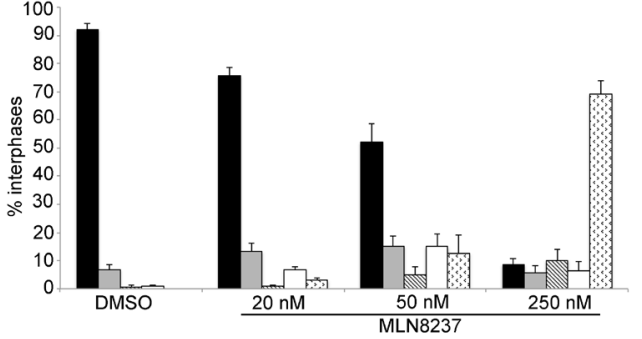

aNormal nuclei $\square$ Chromosome loss/gain

\$PPolyploid $\square$ Binucleated MMultinucleated

Figure 7: Outcome of mitoses treated with the MLN8237 inhibitor. A. Schematization of the protocol for the analysis of the progeny of MLN8237-treated mitoses. B. The IF panels show different scored categories. Markers and color codes for DNA, MTs, nuclear envelope and centrosomes are indicated on the left. In the upper panels, the inset in the DAPI channel shows an enlargement of a micronucleus, while insets in the merged images show enlargements of centrosomes. Scale bars: $10 \mu \mathrm{m}$. C. Histograms represent the occurrence (\%) of defects in B. At least 1000 cells per condition were counted in 3 experiments; s.d. are shown. 
prometaphases from cultures treated with 5,10 or $20 \mathrm{nM}$ MLN8237 compared to controls. The average angle in control prometaphases was $11^{\circ}$ and almost doubled $\left(19^{\circ}\right.$, mean value) in MLN8237-treated cells ( $p<0,01)$, reaching a $>30^{\circ}$ distortion in about $20 \%$ of prometaphases. Thus, the inhibition of Aurora-A under conditions that do not impair spindle formation altogether influences the proper orientation of the spindle axis and hence of cell division.

\section{Induction of aneuploidy in the progeny of MLN8237-treated mitoses}

Some of the defects observed in MLN8237-treated mitoses suggest the possibility that genetically imbalanced daughter cells are generated.

To address this issue we treated pre-synchronized cells with MLN8237 in G2 as described, fixed the cells after 24 hours and screened defects in interphase cells presumably representing the progeny of treated mitoses
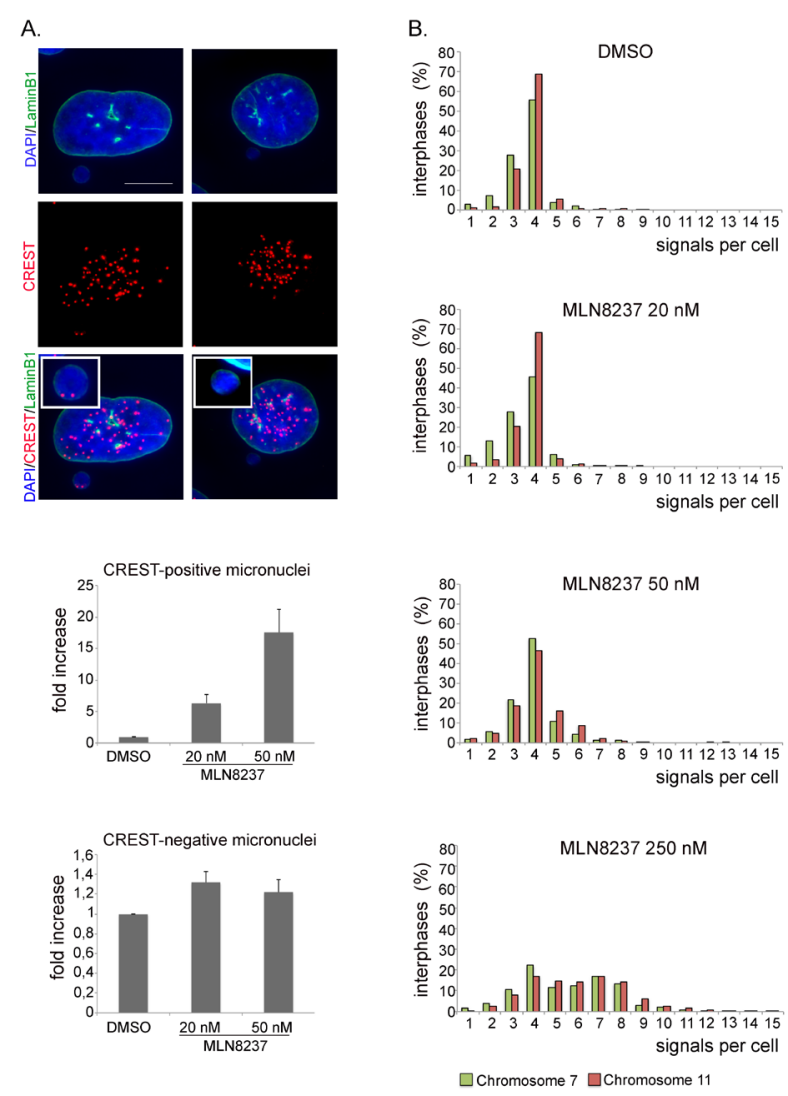

Figure 8: Ploidy alteration in MLN8237-treated cells. A. IF images of CREST-positive (left panels) or -negative (right panels) micronuclei. The insets show enlargements of micronuclei. Histograms represent interphases with CRESTpositive or -negative micronuclei in MLN8237-treated cultures (fold-increase relative to controls). 400 cells per condition were counted from 4 experiments. s.d. are shown. Scale bar: $10 \mu \mathrm{m}$. B. Distribution of interphases with different numbers of signals for chromosomes 7 and 11 (FISH hybridization). 800-1000 cells per condition were counted in 2 experiments.
(Figure 7). Cells were stained with combinations of DAPI and antibodies against alpha-tubulin and pericentrin (examples in Figure 7B, upper panels) or lamin-B1 and alpha- or gamma-tubulin (middle and lower panels) and categorized: cells with loss or gain of 1 or few chromosomes (1-2 micronuclei), polyploid (1 large nucleus with 4 pericentrin or gamma-tubulin signals), binucleated, or multinucleated.

Indeed, following treatment with $250 \mathrm{nM}$ MLN8237, most interphases appeared to have undergone chromosome mis-segregation ( $>65 \%$ multinucleated cells); a smaller fraction (about 10\%) became polyploid. Only less than $10 \%$ of interphases were apparently normal (Figure 7C). Abnormalities were also observed in cells generated during treatment with $50 \mathrm{nM}$ MLN8237: some $20 \%$ were multinucleated or polyploid and about $15 \%$ were binucleated. Interestingly, in about one third of the binucleated cells the nuclei were not equivalent in size, suggesting that they represent aberrant products of the multipolar/asymmetric divisions (see Figure 5). Timelapse imaging of fluorescently labelled U2OS cells showed that some interphases remained very close after division with a connecting alpha-tubulin bridge (Supplementary Figure S4), possibly representing intermediate figures before binucleation.

$20 \mathrm{nM}$ and $50 \mathrm{nM}$ MLN8237 also yielded a significant induction (14-15\%) of cells with micronuclei, indicative of mild aneuploidy (Figure 7C). To assess whether micronuclei reflected genuine chromosome loss events, and hence aneuploidy, we assessed whether they contained whole chromosomes by staining with CREST antibodies to human centromeres (Figure 8A). We observed a 6- and 17-fold increase of CREST-positive micronuclei in 20 and $50 \mathrm{nM}$ MLN8237-treated cultures, respectively, compared to controls. Consistent with this, recording of GFP-labelled chromosomes dynamically visualized chromosome bridges and micronuclei formation; interestingly, these defects were always present in mitoses with multipolar spindles (Supplementary Figure S4).

These results indicate different extents of ploidy alterations in MLN8237-treated cultures, which we decided to investigate directly by employing FISH (Fluorescence In Situ Hybridization) analysis. We counted hybridization signals produced by chromosome-specific centromeric probes (chromosome 7 and 11) in interphases originated from MLN8237-treated mitoses (protocol in Figure 7). Most (about 85\%) control interphases displayed 3-4 signals for both chromosome 7 and 11 (Figure 8B), confirming that U2OS cells are hypertriploid. $20 \mathrm{nM}$ MLN8237 induced a slight shift in the frequency of cells with fewer signals (2-fold increase in interphases with 1-2 signals compared to controls), likely corresponding to the chromosome loss events evidenced by the micronuclei analysis. With $50 \mathrm{nM}$ MLN8237, cells displaying 3-4 signals decreased to about $70 \%$ and cells with $>4$ signals 
correspondingly increased; a minor fraction of truly polyploid cells ( $\geq 6$ signals for both chromosomes within a single large nuclei) was also identified, consistent with the IF results in Figure 7. With 250 nM MLN8237 > 50\% interphases had $\geq 6$ signals, indicative of gain of complete sets of chromosomes, consistent with the lack of division observed in time-lapse experiments (Figure 5) and with the frequent multinucleated state depicted in Figure 7. FACS analysis confirmed the increase in ploidy in about $45 \%$ of the viable population (data not shown) after 48 and 96 hours of treatment with $250 \mathrm{nM}$ MLN8237.

Together these analyses indicate that MLN8237 treatment yields variable levels of aneuploidy in daughter cells, in a dose-dependent manner.

\section{Long term fate of MLN8237-treated cells}

The time-lapse analysis thus far indicates that MLN8237 (5-250 nM range) induces no significant cell death in U2OS mitotic cells. We investigated the longterm outcome of the treatment in high-throughput timelapse analyses for a length of time (48 hours) roughly corresponding to 2 division cycles. Using the fluorescently labelled U2OS cell line, we analyzed the data with an automated method, the CellCognition software [28], trained to classify cells as interphasic, mitotic, dead, multinucleated or polyploid (examples in Figure 9A). In the 48 hours of the recording time, the cell number increased threefold in control cultures, yet dose-dependent growth inhibition was observed in cultures treated with MLN8237, with almost no increase with $250 \mathrm{nM}$ MLN8237 (Figure 9B, left panel). Consistent with this, the number of normal interphase cells was dramatically reduced by the treatment (Figure 9B, right panel): this effect could result either from an increase in mitotic cells (due to prolonged mitotic

Figure 9: Long-term high-throughput analysis of MLN8237-treated cultures. Cultures treated as in Fig. 3 were recorded for 48 hours. Automated segmentation and classification on the images was performed using the CellCognition software. A. Classes defined for training the classifier are shown with representative examples for each class. B. Histograms on the left represent the increase in the number of viable cells from the first to the last frame of the acquisition. Average values and s.d. of 10 replicates from 3 independent experiments are shown. The analyzed sample size at time 0 was at least 500 cells per replicate. Histograms on the right represent the increase in normal interphases only under the same conditions. C. The line charts represent the percentage of cells per class during the recording time.

A. duration) or from the generation of abnormal interphase cells. The kinetics of appearance of mitotic cells depicted two waves of division in control cultures (Figure 9C). In MLN8237-treated cultures the first mitotic peaks were shifted in time and appeared broader. Both effects were dose-dependent, consistent with our data on mitotic entry and duration (Figures 2 and 3). At $250 \mathrm{nM}$ inhibitor, no second wave of division was observed. Concomitantly, we observed a strong increase of multinucleated cells at $250 \mathrm{nM}$ and a milder effect at $50 \mathrm{nM}$ MLN8237. Some polyploid cells appeared under these conditions (below $3 \%$ ). Detection of multinucleated and polyploid cells was therefore consistent with the results from fixed samples (Figures 7 and 8). Importantly, the induction of cell death remained below 3\% throughout the recording time (Figure $9 \mathrm{C}$, bottom right panel), and remained at a similar level in one time-lapse experiment extended to 65 hours (not shown).

Together, the high-throughput data indicate that MLN8237 induces a dose-dependent lengthening of mitotic progression and the generation of abnormal daughter cells that are impaired in re-dividing, with a cytostatic effect, while low cytotoxicity is observed within the first 48 hours of treatment.

We also performed proliferation/cell death analyses

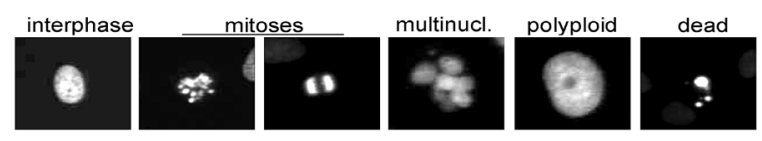

B.
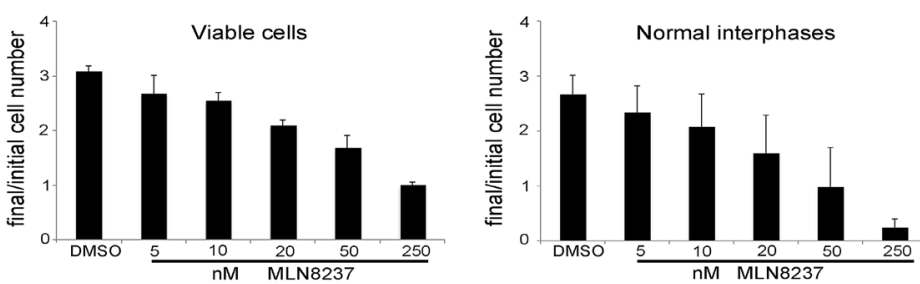

C.
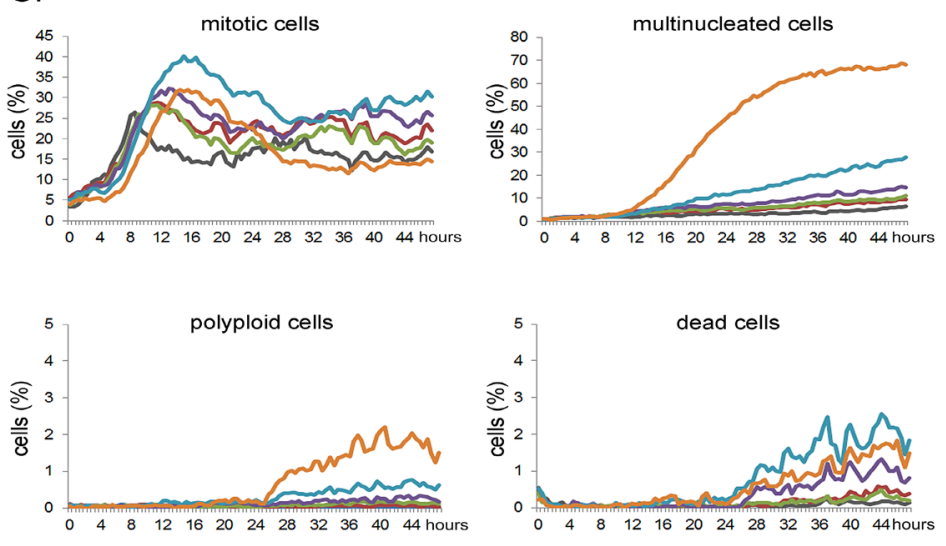

-DMSO

$=5 \mathrm{nM}=10 \mathrm{nM} \rightleftharpoons 20 \mathrm{nM}=50 \mathrm{nM}=250 \mathrm{nM}$ 
after several days (Figure 10) by cell counting and FACS measurement of the DNA content. After 48 hours of treatment, corresponding to the end of the high-throughput video-recording, a slight decrease in the total cell number was observed with 5-10-20 nM MLN8237 compared to control cultures (Figure 10A); the overall proliferation trend remained comparable to controls over 96 hours. Cell growth inhibition was instead appreciated with 50 nM MLN8237 and even more effectively with $250 \mathrm{nM}$ MLN8237 (Figure 10A).

To assess cell death we measured cells with a sub-G1 DNA content by FACS analysis (Figure 10B). That revealed a generally higher level of cell death compared to the microscopy analysis, possibly reflecting technical specificities in the methodology: indeed, detached dead cells are counted by cytofluorimetry, while being preferentially lost in microscopy analysis. Nevertheless, by FACS analysis, only $250 \mathrm{nM}$ MLN8237 induced remarkable cell death (about $30 \%$ of sub-G1 cells after 48 hours of treatment, increasing to about $50 \%$ after 96 hours, compared to about $10 \%$ in control cultures; Figure 10B).

Thus, MLN8237 treatment in concentrations that

A.

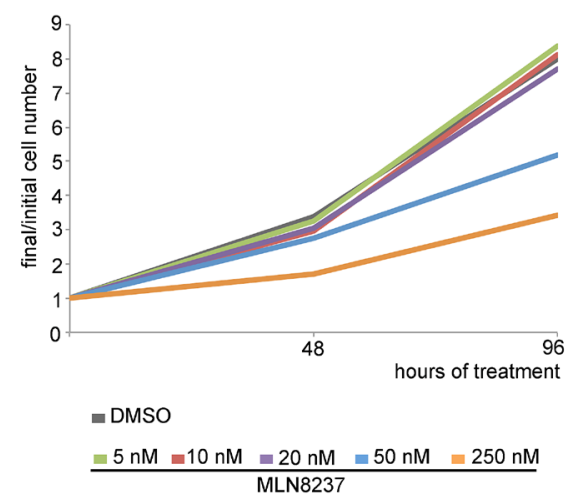

B.

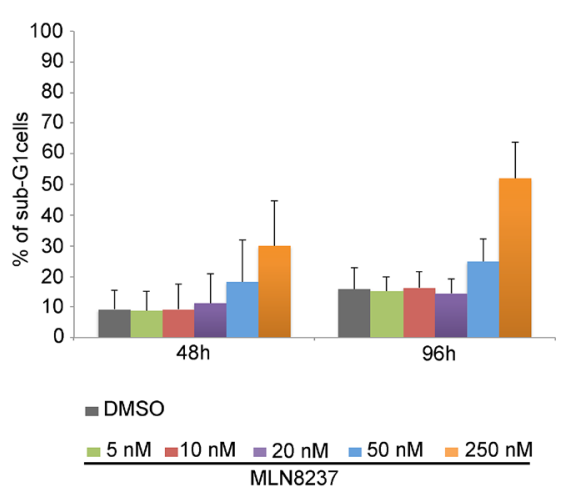

Figure 10: Dose-dependent effects of MLN8237 on cell growth and viability. A. Cells were counted after 48 and 96 hours from MLN8237 treatment start. Values represent the increase in the number of cells respect to $\mathrm{t}=0 \mathrm{~h}$ ( 3 independent experiments). B. The percentage of sub-G1 cells in MLN8237treated cultures, detected by FACS analysis, is shown in the histograms (3 experiments). s.d. are shown. genuinely inhibit Aurora-A does not efficiently promote cell death in U2OS cells; a toxic effects is only observed after very long exposure times to high doses that simultaneously target Aurora-B.

\section{DISCUSSION}

Here we have used the MLN8237 small molecule inhibitor to investigate mitotic roles of the Aurora-A kinase in osteosarcoma U2OS cells; this molecule is under clinical trial in several cancer types including osteosarcoma.

We first determined the extent and specificity of kinase inhibition by assessing Aurora-A and Aurora-B self-phosphorylation. MLN8237 was effective over Aurora-A in the $20-50 \mathrm{nM}$ range, but also inhibited Aurora-B above $50 \mathrm{nM}$. The loss of selectivity with high doses was previously reported [25-27]. The inhibition of Aurora-B identified here with 50 nM MLN8237 (a very close condition to that required for complete Aurora-A inhibition) was instead not noticed before using histone $\mathrm{H} 3$ phosphorylation as a reporter, indicating that the selection of activity reporters is critical to ascertain the selectivity of kinase inhibitors. It also evidences that the window at which MLN8237 fully inhibits Aurora-A, without concomitantly affecting Aurora-B, can be very narrow in some cell lines, indicating that selective inhibition of Aurora-A vs Aurora-B remains a critical issue, even with the best performing ATP-competitors.

MLN8237 treatment prolongs the G2 phase duration. The $\mathrm{G} 2$ delay was under-appreciated in previous studies using MLN8237 in asynchronous cultures, yet was observed when Aurora-A was inhibited by either antibody microinjection or RNA interference [1, 29], or by conditional ablation in mouse embryonic fibroblasts (MEFs; [30]). No permanent arrest is however induced, suggesting that Aurora-A functions in G2 are important, but can be taken over by other kinases. Indeed, the G2 delay is more severe with $250 \mathrm{nM}$, under which condition Aurora-B is also inhibited. These findings raise the possibility that centrosome- and/or MT-associated defects induced by Aurora-A inactivation in G2 evoke a checkpoint response that delays the transition towards mitosis onset.

Dose-response analyses of MLN8237-treated cells that entered mitosis revealed processes that are differentially sensitive to Aurora-A inactivation, as depicted in the schematics in Figure 11. Complete Aurora-A inhibition impaired MT nucleation; consistent with this, previous data showed that partial vs complete RNA interference-mediated depletion of Aurora-A differentially affects maturation of centrosomes, required for mitotic MT nucleation [1, 2]. Partial Aurora-A inhibition (10 nM MLN8237) instead yielded multipolar or disorganized spindles, the frequency of which increased in a dose-dependent manner up to $50 \mathrm{nM}$. 
Defective spindle assembly was associated with longer prometaphase duration compared to untreated cells. The highest frequency of induction of spindle organization defects $(50 \mathrm{nM})$ was associated with multipolar divisions.

$250 \mathrm{nM}$ MLN8237 also prolonged prometaphase duration, after which cells re-adhered and eventually exited mitosis without division. The failure of chromosome segregation could be appreciated in timelapse experiments and was consistent with previous results obtained from concomitant inactivation of Aurora-A and B kinases by high MLN8237 doses in Hep3B or HeLa cells or, to a lesser extent, following Aurora-B inhibition in HeLa cells $[26,27]$. Our own recording experiments of interfered mitoses for Aurora-A, or -B, or both, confirm a mild effect of Aurora-B inactivation alone and a synergic effect of the inactivation of both kinases. Complementary functions of Aurora-A and B in chromosome segregation also emerged in chicken DT40 Aurora- $\mathrm{A}^{\mathrm{KO}}$ cells treated with an Aurora-B specific inhibitor [31]. None of the approaches used to inactivate Aurora-A alone in human cultured cells yielded chromosome segregation failure [2, 27, 32]. The latter was instead described in Aurora-A-null MEFs [30, 33], suggesting specific modes of action of the two kinases in this cellular context, which may reflect a different stoichiometry between Aurora-A and B and/or their substrates.

An interesting finding from this study is the induction of mis-oriented divisions by low MLN8237 concentrations $(5-20 \mathrm{nM})$; the inhibitor is selective for Aurora-A at these doses and, as recalled above, does not impair MT nucleation: this directly implicates the lack of Aurora-A in the mis-orientation phenotype and suggests that MTs are required. Interestingly, Aurora-A

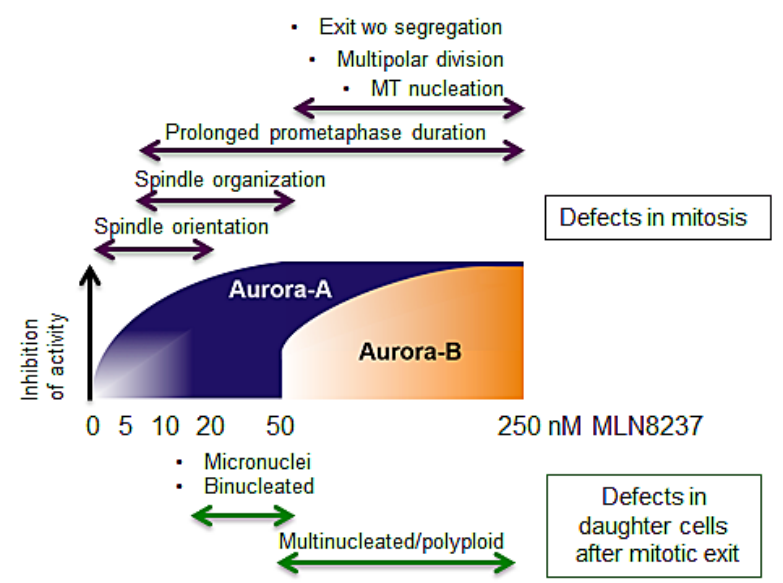

Figure 11: Dose-dependent effects of MLN8237 on cell division and aneuploidy induction: a schematic overview. Schematic representation of the phenotype distribution (Up: mitotic defects. Low: abnormal daughter interphases) along a MLN8237 concentration gradient in U2OS cells. Gradual inhibition of Aurora-A (blue) and Aurora-B (orange) is indicated by the gradients within shapes (dose intervals are not in scale); note that at 20 nM MLN8237 Aurora-A inhibition is virtually complete. is implicated in spindle orientation in asymmetric cell divisions in Drosophila [34] via phosphorylation of Pins [35]. Excess Aurora-A can also influence the spindle orientation and the cell fate in human mammary epithelium stem/progenitor cells [36]. In our time-lapse assays using a U2OS cell line with fluorescent MTs, we actually visualized spindle rotation movements: thus, Aurora-A activity is required for pathways that determine the mitotic spindle orientation, raising the possibility that Aurora-A inhibition influences the fate of asymmetrically dividing cells and/or tissue architecture.

Time-lapse experiments also revealed that MLN8237 generates chromosome mis-segregation. At high concentrations, when both Aurora-A and Aurora-B are inhibited, massive aneuploidy is observed, with the generation of multinucleated daughter cells. Lower doses that inhibit Aurora-A alone yield mild aneuploidy, with increased number of CREST-positive micronuclei (schematics in Figure 11). FISH analysis depicted the differential generation of mild and massive aneuploidy by low and high MLN8237 doses, respectively. This is relevant, given the pro- or anti-tumorigenic effects of aneuploidy depending on the extent of chromosome missegregation [24]. Consistent with our observations, Yang and colleagues [37] described the generation of an $8 \mathrm{~N}$ population following simultaneous RNA interferencemediated inactivation of Aurora-A and Aurora-B, but not of Aurora-A alone, in U2OS cells. Segregation defects (lagging chromosomes and chromatin bridges) were observed in Aurora-A-null MEFs, with increased ploidy over time [30,33], again evidencing a crucial contribution of Aurora-A to chromosome segregation in this system.

We also observed binucleated cells in the progeny of MLN8237-treated mitoses; in time-lapse analyses, telophase cells often remained in closer proximity to one another compared to controls, and tubulin bridges persisted, a phenotype that may possibly evolve into a binucleated cell. The observation of binucleated cells with 20 nM MLN8237 would support a recently proposed direct role of Aurora-A in late steps of cell division [6, 7].

Importantly, MLN8237 failed to induce cell death in mitosis nor did it cause a highly effective elimination of the cellular offspring within the first 48 hours of treatment. Recent time-lapse studies with MLN8237 reported variable results regarding mitotic cell death: no mitotic toxicity was observed in Hep3B cells [26], whereas some death from mitosis was recorded in HeLa cells [27]. Aurora-A inactivation therefore appears to require a set of concomitant conditions, as yet elusive, for mitotic cell death activation. The highly aneuploid progeny generated in our assays at $50 \mathrm{nM}$ (and, to a higher extent, $250 \mathrm{nM}$ ) MLN8237 originates cells impaired in further cell division, hence unviable in the long term. The mechanisms underlying the cytostatic effects of Aurora-A inactivation are controversial: a dose- and time-dependent induction of apoptosis was described in different cell 
lines treated with MLN8237 [18, 19, 38, 39], while in other cases the MLN8237-induced cytostatic effect is attributed to senescence [40] consistent with results described in Aurora-A-null MEFs [33], or to induction of differentiation pathways [41]. These observations suggest that both the treatment parameters and the cellular background contribute to determine the long-term outcome of MLN8237-treated cultures.

In conclusion, the broad variability in the U2OS cell response to MLN8237 highlighted in this study is an important issue in the light of the use of this compound in anti-cancer therapy. In the human organism undergoing treatment, the compound dose cannot be constant and is expected to rise and fall over time. It will be important to extend these studies and shed light on the pathways driving the response towards one or another cell fate, with the perspective to modulate such choice and drive cells towards death pathways.

\section{METHODS}

\section{Cell cultures, synchronization protocols and treatments}

The human U2OS osteosarcoma cell line (ATCC: HTB-96) was grown at $37^{\circ} \mathrm{C}$ in a $5 \% \mathrm{CO}_{2}$ atmosphere in DMEM, supplemented with $10 \%$ faetal bovine serum. U2OS cells stably expressing H2B-GFP and RFP-alphatubulin (kind gift of L. Lanzetti, Institute for Cancer Research at Candiolo, Italy) were cultured under the same conditions and used in time-lapse imaging experiments. For synchronization, cells were subjected to a 24 hours block in $2 \mathrm{mM}$ thymidine. Cultures were then released from the G1/S arrest by washing away the thymidine and adding fresh medium containing $30 \mu \mathrm{M}$ deoxycytidine; 6 hours post-release cultures were treated with MLN8237 (5-250 $\mathrm{nM}$ as indicated; Selleck Chemicals) or $0.1 \mu \mathrm{g} / \mathrm{ml}$ nocodazole (Sigma Aldrich). Mock-treated cultures were incubated with DMSO.

\section{Cell counting and FACS analysis}

Cells were harvested after 48 and 96 hours from treatment. One sample was harvested when cultures were released in thymidine-free medium $(\mathrm{t}=0 \mathrm{~h})$ as reference for the initial number of cells and for verifying the efficacy of the thymidine arrest. For counting the number of cells a Z1 Coulter Particle Counter (Beckman Coulter) was used. For FACS analysis samples were permeabilized with $0.1 \%$ TritonX-100 in PBS. Cell cycle phase distribution was analyzed after incubation with propidium iodide (PI, Sigma P4170, $0.04 \mathrm{mg} / \mathrm{ml}$ ) using a flow cytofluorimeter Epics XL apparatus (Beckman Coulter). Parameters SS and FL-3 were acquired in a linear amplification scale,
FS and FL-2, in a log scale. Cell aggregates were gated out on the bi-parametric graph FL-3lin/Ratio. Apoptosis was determined as the proportion of cells exhibiting a DNA content lower than that of G1 cells after gating out cell debris on the bi-parametric graph FS/SS, using the WinMDI software.

IF

Cells grown on coverslips were fixed as follows: (a) $-20^{\circ} \mathrm{C}$ methanol, 6 minutes; or, for phosphoAurora-B (Thr232) staining, (b) 3.7\% PFA in PBS, 10 minutes at room temperature followed by 5 minutes in $0.1 \%$ TritonX-100 in PBS. Blocking and all antibody incubations were performed at room temperature in PBS $/ 0.05 \%$ Tween 20/3\% BSA. Cells were counterstained with 4,6-diamidino-2-phenylindole (DAPI, $0.1 \mu \mathrm{g} / \mathrm{ml}$ ) and mounted using Vectashield (Vector Laboratories). Primary antibodies were: mouse anti-alpha-tubulin (1:2000, B-5-12, Sigma-Aldrich), mouse anti-Aurora-A (0.5 $\mu \mathrm{g} / \mathrm{ml}, \mathrm{BD}$ Transduction Laboratories), rabbit anti-phospho-Aurora-A (Thr288) (1:250, C39D8; Cell Signaling Technology), rabbit anti-pericentrin $(2 \mu \mathrm{g} / \mathrm{ml}$, ab4448; Abcam $)$, rabbit anti-phospho-Aurora-B (Thr232) (1:50, Poly6361, BioLegend), mouse anti-phospho-Histone-H3 (Ser 10) $(0.25 \mu \mathrm{g} / \mathrm{ml}$, clone $3 \mathrm{H} 10$, Millipore $)$, mouse anti- $\gamma$-tubulin (1:1000, GTU-88, Sigma-Aldrich), rabbit anti-lamin B1 (1 $\mu \mathrm{g} / \mathrm{ml}$, ab16048; Abcam), human anti-centromere (CREST; 1:20, Antibodies Incorporated). Samples were analyzed using a Nikon Eclipse 90i microscope equipped with a Qicam Fast 1394 CCD camera (QImaging). Image acquisition, deconvolution and Extended Depth of Focus on Z-serial optical sections were performed using NisElements AR 4.2 (Nikon); images were further processed with Adobe Photoshop CS 8.0.

\section{Quantitative analysis of IF signals.}

Signals were measured using Nis Elements AR 4.2 (nd2 file format). Analysis of endogenous Aurora-A and Aurora-B activity in mitotic cells was performed as follows: a) p-Thr288-Aurora-A staining: average pixel intensity at spindle poles, corrected for external background; b) p-Thr232-Aurora-B staining: sum intensity at chromosomes, corrected for external background. Images for quantification of mitotic signals were Maximum Intensity Projections from z-stacks $(0.6 \mu \mathrm{m}$, ranging over a 5-10 $\mu \mathrm{m}$ ). Box-plots were generated using the web-tool BoxPlotR.

For measuring the angle between the centrosomecentrosome axis and the culture surface z-stacks serial images were used. The " $\arctan (\mathrm{xy} / \mathrm{z})$ " formula for calculating the angle of a right triangle was applied with "xy" being the distance between centrosomes in xy in the maximum intensity projection, and " $z$ " being the distance 
between centrosomes along the $\mathrm{z}$-axis. A schematization is shown in Figure 6C. Values were statistically analyzed using the InStat 3 software, using either (i) the unpaired $\mathrm{t}$ test (for Gaussian distributions), applying the Welch correction when required, or (ii) the Mann-Whitney test, when the populations did not follow a Gaussian distribution.

\section{Fluorescent in situ hybridization (FISH)}

Cells grown on coverslips were fixed at room temperature with 3:1 methanol:acetic acid, air dried and stored at $-20^{\circ} \mathrm{C}$ for at least one day. Before hybridization, coverslips were again incubated in 3:1 methanol:acetic acid, 1 hour at room temperature, then heated 2 hours at $65^{\circ} \mathrm{C}$ and dehydrated in $70 \%-90 \%-100 \%$ cold ethanol. Denaturation of probes in the hybridization mix (Fluorescein-labeled Chromosome 7 Satellite Probe, cat: PSAT0007-G; rhodamine-labeled Chromosome 11 Satellite Probe, cat: PSAT001-R; Hybridization Buffer QB007, all from Q-BIOgene) was performed at $96^{\circ} \mathrm{C}$ for 10 minutes. When the mix was applied on coverslips a co-denaturation step was performed ( 2 minutes, $72{ }^{\circ} \mathrm{C}$ ), followed by the hybridization incubation, performed at $37^{\circ} \mathrm{C}$, overnight. Coverslips were then washed in SSC, at $37^{\circ} \mathrm{C}$ and $60^{\circ} \mathrm{C}$, and counterstained with DAPI $(0.2 \mu \mathrm{g} /$ $\mathrm{ml})$ in SSC, 10 minutes at room temperature. Coverslips were mounted with Vectashield and analyzed with a Nikon Eclipse 90i microscope, using a 20x objective (Plan Fluor, 0.5 N.A.). Signals per nucleus were counted using the Object count function of Nis Elements AR 4.2 (nd2 file format), setting the parameters using the Spot detection function.

\section{Time-lapse recording}

Cells seeded in $35 \mathrm{~mm}$ dishes (ibiTreat, cod. 81156, or glass bottom, cod. 81158 , both from Ibidi) or 8-well micro-slides (ibiTreat, cod. 80826, Ibidi) were observed under an Eclipse Ti inverted microscope (Nikon), using 60x [Plan Apo, 1.4 N.A. Differential Interference Contrast (DIC), oil immersion] or 40x (Plan Fluor, 0.60 N.A. DIC) objectives (Nikon); during the whole observation cells were kept in a microscope stage incubator (Basic WJ, Okolab), at $37^{\circ} \mathrm{C}$ and $5 \% \mathrm{CO}_{2}$. DIC images were acquired every 5 minutes over 24 hours using a DS-Qi1Mc camera and the NIS-Elements AR 3.22 software (Nikon). Image and movie processing were performed with NIS-Elements AR 4.2.

Imaging of the U2OS cell line stably expressing H2B-GFP and RFP-alpha-tubulin was performed with the 60x objective: images were acquired every 7 minutes in the 3 channels. $z$-stacks of the fluorescent channels were acquired every $1 \mu \mathrm{m}$ over a range of $8 \mu \mathrm{m}$, attenuating the fluorescence lamp intensity to $1 / 32$. Under these conditions, time-lapse acquisition, starting 6 hours after the treatment, was extended for 8 hours only to avoid phototoxic effects.

For high-throughput experiments, cells were seeded in 96-well plates. Images were acquired with a ScanR microscope (Olympus) using a 10x objective, an Olympus DBH1 camera and the ScanR acquisition software. Temperature and $\mathrm{CO}_{2}$ were kept constant by an incubation system on the microscope. Acquisition was performed every 30 minutes using Phase contrast and fluorescence imaging, for a total duration of 48-65 hours.

\section{Automated analysis of high-throughput data}

Data from high-throughput experiments were analyzed by the CellCognition software (v 1.3.3-28, [28]). The software was used to segment the cells, extract features and classify them using a support vector machine. The classes defined were: interphases, prometaphases, metaphases, ana-telophases, multinucleated interphases, poliployd cells, dead cells; an additional class of debris or of cells with non homogeneous morphology which could not be included in any of the other categories was created and was then excluded from subsequent data elaboration to avoid artifacts. The classifier was trained with images from independent experiments with a training set comprising a minimum of 100 cells per class (with the exception of the polyploid class, of which only 33 examples were found). A confusion matrix and a classification test were used to assess the quality of classification before the analysis. The output data were further analyzed using Microsoft Excel; a single class for Mitoses was generated by pooling the prometaphase, metaphase and ana-telophase classes. Values for each well were pooled from 6 imaging fields; average values from 4 replicates within one experiment are shown in Figure 9. A similar trend was observed in 2 independent experiments (6 additional replicates).

\section{Immunoblotting}

Mitotic cells collected by shake off were lysed in RIPA buffer (50 mM Tris- $\mathrm{HCl} \mathrm{pH} 8.0,150 \mathrm{mM} \mathrm{NaCl}$, $1 \%$ NP40, 1 mM EGTA, 0.25\% sodium deoxycholate) supplemented with protease and phosphatase inhibitors. Proteins were resolved by electrophoresis on $10 \%$ Laemmli gel and transferred on a nitrocellulose membrane (Protran BA83, Whatman) using a semi-dry system (BIORAD). $40 \mu \mathrm{g}$ of extract per lane were loaded. Blocking and antibody incubations were performed at room temperature in $\mathrm{PBS} / 0.1 \%$ Tween $20 / 5 \%$ low fat milk, or in PBS/0.1\% Tween 20/5\% BSA (for anti-phosphoAurora-A hybridization). Antibodies were: mouse antiAurora-A ( $1 \mu \mathrm{g} / \mathrm{ml}$; BD Transduction Laboratories), rabbit anti-phospho-Aurora-A (Thr288) (1:1000; C39D8; Cell Signaling Technology), goat anti-actin $(0.5 \mu \mathrm{g} / \mathrm{ml}, \mathrm{I}-19$; 
SantaCruz Biotechnology). Signals were visualized by enhanced chemiluminescence detection (ECL plus, GE Healthcare, and Protein Detection System, GeneSpin).

\section{ACKNOWLEDGEMENTS}

We are grateful to Dr. Rainer Pepperkok and the EuroBioImaging project for access to the Advanced Light Microscopy Facility of the EMBL (Heidelberg) and to the team at the Facility for advice and technical help. We thank Dr. Letizia Lanzetti for providing the U2OS cell line stably expressing fluorescent GFP-H2B and RFP-alpha-tubulin and Dr. Francesca Degrassi for reagents and advice with FISH analysis. We are grateful to Dr. Pietro Cirigliano and to staff of Nikon Instruments S.p.A. (Italy) for continuous collaborative support for imaging experiments performed at the CNR-IBMN Nikon Reference Center. This work was supported by grants from AIRC-Italian Association for Cancer Research (MFAG-13350 to G.G. and IG14534 to P.L.) and Fondazione Monte dei Paschi di Siena (to G.G. and P.L.). Visits of IAA to the ALMF were supported by an EMBO Short-term fellowship and a SIBBM travel grant.

\section{REFERENCES}

1. Hirota T, Kunitoku N, Sasayama T, Marumoto T, Zhang D, Nitta M Hatakeyama K, Saya H. Aurora-A and an interacting activator, the LIM protein Ajuba, are required for mitotic commitment in human cells. Cell. 2003; 114: 585-598

2. Marumoto T, Honda S, Hara T, Nitta M, Hirota T, Kohmura E, Saya H. Aurora-A kinase maintains the fidelity of early and late mitotic events in HeLa cells. J Biol Chem. 2003; 278: 51786-51795.

3. Katayama H, Sasai K, Kloc M, Brinkley BR, Sen S. Aurora kinase-A regulates kinetochore/chromatin associated microtubule assembly in human cells. Cell Cycle. 2008; 7 : 2691-2704.

4. De Luca M, Lavia P, Guarguaglini G. A functional interplay between Aurora-A, Plk1 and TPX2 at spindle poles: Plk1 controls centrosomal localization of Aurora-A and TPX2 spindle association. Cell Cycle. 2006; 5: 296-303.

5. De Luca M, Brunetto L, Asteriti IA, Giubettini M, Lavia P, Guarguaglini G. Aurora-A and ch-TOG act in a common pathway in control of spindle pole integrity. Oncogene. 2008; 27: 6539-6549.

6. Lioutas A, Vernos I. Aurora A kinase and its substrate TACC 3 are required for central spindle assembly. EMBO Rep. 2013; 14: 829-836.

7. Reboutier D, Troadec MB, Cremet JY, Chauvin L, Guen V, Salaun P, Prigent C. Aurora A is involved in central spindle assembly through phosphorylation of Ser 19 in P150Glued. J Cell Biol. 2013; 201: 65-79.

8. Carmena M, Wheelock M, Funabiki H, Earnshaw WC. The chromosomal passenger complex (CPC): from easy rider to the godfather of mitosis. Nat Rev Mol Cell Biol. 2012; 13: 789-803.

9. Lapenna S, Giordano A. Cell cycle kinases as therapeutic targets for cancer. Nat Rev Drug Discov. 2009; 8: 547-566.

10. Lens SM, Voest EE, Medema RH. Shared and separate functions of polo-like kinases and aurora kinases in cancer. Nat Rev Cancer. 2010; 10: 825-841.

11. Kollareddy M, Zheleva D, Dzubak P, Brahmkshatriya PS, Lepsik M, Hajduch M. Aurora kinase inhibitors: progress towards the clinic. Invest New Drugs. 2012; 30: 2411-2432.

12. Cervantes A, Elez E, Roda D, Ecsedy J, Macarulla T, Venkatakrishnan K, Roselló S, Andreu J, Jung J, SanchisGarcia JM, Piera A, Blasco I, Maños L, et al. Phase I pharmacokinetic/pharmacodynamic study of MLN8237, an investigational, oral, selective aurora a kinase inhibitor, in patients with advanced solid tumors. Clin Cancer Res. 2012; 18: 4764-4774.

13. Dees EC, Cohen RB, von Mehren M, Stinchcombe TE, Liu H, Venkatakrishnan K, Manfredi M, Fingert H, Burris HA 3rd, Infante JR. Phase I study of aurora A kinase inhibitor MLN8237 in advanced solid tumors: safety, pharmacokinetics, pharmacodynamics, and bioavailability of two oral formulations. Clin Cancer Res. 2012; 18: 47754784.

14. Matulonis UA, Sharma S, Ghamande S, Gordon MS, Del Prete SA, Ray-Coquard I, Kutarska E, Liu H, Fingert H, Zhou X, Danaee H, Schilder RJ. Phase II study of MLN8237 (alisertib), an investigational Aurora A kinase inhibitor, in patients with platinum-resistant or -refractory epithelial ovarian, fallopian tube, or primary peritoneal carcinoma. Gynecol Oncol. 2012; 127: 63-69.

15. Doménech E, Malumbres M. Mitosis-targeting therapies: a troubleshooting guide. Curr Opin Pharmacol. 2013; 13: 519-528.

16. Friedberg JW, Mahadevan D, Cebula E, Persky D, Lossos I, Agarwal AB, Jung J, Burack R, Zhou X, Leonard EJ, Fingert H, Danaee H, Bernstein SH. Phase II study of alisertib, a selective Aurora A kinase inhibitor, in relapsed and refractory aggressive B- and T-cell non-Hodgkin lymphomas. J Clin Oncol. 2014; 32: 44-50.

17. Manfredi MG, Ecsedy JA, Chakravarty A, Silverman L, Zhang M, Hoar KM, Stroud SG, Chen W, Shinde V, Huck JJ, Wysong DR, Janowick DA, Hyer ML et al. Characterization of Alisertib (MLN8237), an investigational small-molecule inhibitor of aurora A kinase using novel in vivo pharmacodynamic assays. Clin Cancer Res. 2011; 17: 7614-7624.

18. Kelly KR, Nawrocki ST, Espitia CM, Zhang M, Yang JJ, Padmanabhan S, Ecsedy J, Giles FJ, Carew JS. Targeting Aurora A kinase activity with the investigational agent alisertib increases the efficacy of cytarabine through a FOXO-dependent mechanism. Int J Cancer. 2012; 131: 2693-2703. 
19. Qi W, Spier C, Liu X, Agarwal A, Cooke LS, Persky DO, Chen D, Miller TP, Mahadevan D. Alisertib (MLN8237) an investigational agent suppresses Aurora A and B activity, inhibits proliferation, promotes endo-reduplication and induces apoptosis in T-NHL cell lines supporting its importance in PTCL treatment. Leuk Res. 2013; 37: 434439.

20. Maris JM, Morton CL, Gorlick R, Kolb EA, Lock R, Carol H, Keir ST, Reynolds CP, Kang MH, Wu J, Smith MA, Houghton PJ. Initial testing of the aurora kinase A inhibitor MLN8237 by the Pediatric Preclinical Testing Program (PPTP). Pediatr Blood Cancer. 2010; 55: 26-34.

21. Zhou N, Singh K, Mir MC, Parker Y, Lindner DJ, Dreicer R, Ecsedy JA, The BT, Zhang Z, Almasan A, Hansel DE. The investigational Aurora kinase A inhibitor MLN8237 induces defects in cell viability and cell-cycle progression in malignant bladder cancer cells in vitro and in vivo. Clin Cancer Res. 2013; 19: 1717-1728.

22. Gascoigne KE, Taylor SS. Cancer cells display profound intra- and interline variation following prolonged exposure to antimitotic drugs. Cancer Cell. 2008; 14: 111-122.

23. Asteriti IA, Giubettini M, Lavia P, Guarguaglini G. Aurora-A inactivation causes mitotic spindle pole fragmentation by unbalancing microtubule-generated forces. Mol Cancer. 2011; 10: 131.

24. Weaver BA, Cleveland DW. The aneuploidy paradox in cell growth and tumorigenesis. Cancer Cell. 2008; 14: 431-433.

25. Qi W, Cooke LS, Liu X, Rimsza L, Roe DJ, Manziolli A, Persky DO, Miller TP, Mahadevan D. Aurora inhibitor MLN8237 in combination with docetaxel enhances apoptosis and anti-tumor activity in mantle cell lymphoma. Biochem Pharmacol. 2011; 81: 881-890.

26. Marxer M, Foucar CE, Man WY, Chen Y, Ma HT, Poon RY. Tetraploidization increases sensitivity to Aurora B kinase inhibition. Cell Cycle. 2012; 11: 2567-2577.

27. Marxer M, Ma HT, Man WY, Poon RY. p53 deficiency enhances mitotic arrest and slippage induced by pharmacological inhibition of Aurora kinases. Oncogene. 2014; 33: 3550-3560.

28. Held M, Schmitz MH, Fischer B, Walter T, Neumann B, Olma MH, Peter M, Ellenberg J, Gerlich DW. CellCognition: time-resolved phenotype annotation in highthroughput live cell imaging. Nat Methods. 2010; 7: 747754.

29. Marumoto T, Hirota T, Morisaki T, Kunitoku N, Zhang D, Ichikawa Y, Sasayama T, Kuninaka S, Mimori T, Tamaki N, Kimura M, Okano Y, Saya H. Roles of aurora-A kinase in mitotic entry and G2 checkpoint in mammalian cells. Genes Cells. 2002; 7: 1173-1182.

30. Cowley DO, Rivera-Pérez JA, Schliekelman M, He YJ, Oliver TG, Lu L, O’Quinn R, Salmon ED, Magnuson T, Van Dyke T. Aurora-A kinase is essential for bipolar spindle formation and early development. Mol Cell Biol. 2009; 29: 1059-1071.
31. Hégarat N, Smith E, Nayak G, Takeda S, Eyers PA, Hochegger H. Aurora A and Aurora B jointly coordinate chromosome segregation and anaphase microtubule dynamics. J Cell Biol. 2011; 195: 1103-1113.

32. Du J, Hannon GJ. Suppression of p160ROCK bypasses cell cycle arrest after Aurora-A/STK15 depletion. Proc Natl Acad Sci U S A. 2004; 101: 8975-8980.

33. Pérez de Castro I, Aguirre-Portolés C, Fernández-Miranda G, Cañamero M, Cowley DO, Van Dyke T, Malumbres M. Requirements for Aurora-A in tissue regeneration and tumor development in adult mammals. Cancer Res. 2013; 73: 6804-6815.

34. Lee CY, Andersen RO, Cabernard C, Manning L, Tran KD, Lanskey MJ, Bashirullah A, Doe CQ. Drosophila Aurora-A kinase inhibits neuroblast self-renewal by regulating aPKC/ Numb cortical polarity and spindle orientation. Genes Dev. 2006; 20: 3464-3474.

35. Johnston $\mathrm{CA}$, Hirono $\mathrm{K}$, Prehoda KE, Doe $\mathrm{CQ}$. Identification of an Aurora-A/PinsLINKER/Dlg spindle orientation pathway using induced cell polarity in S2 cells. Cell. 2009; 138: 1150-1163.

36. Regan JL, Sourisseau T, Soady K, Kendrick H, McCarthy A, Tang C, Brennan K, Linardopoulos S, White DE, Smalley MJ. Aurora A kinase regulates mammary epithelial cell fate by determining mitotic spindle orientation in a Notch-dependent manner. Cell Rep. 2013; 4: 110-123.

37. Yang H, Burke T, Dempsey J, Diaz B, Collins E, Toth J, Beckmann R, Ye X. Mitotic requirement for aurora A kinase is bypassed in the absence of aurora B kinase. FEBS Lett. 2005; 579: 3385-3391.

38. Kelly KR, Ecsedy J, Medina E, Mahalingam D, Padmanabhan S, Nawrocki ST, Giles FJ, Carew JS. The novel Aurora A kinase inhibitor MLN8237 is active in resistant chronic myeloid leukaemia and significantly increases the efficacy of nilotinib. J Cell Mol Med. 2011; 15: 2057-2070.

39. Sehdev V, Peng D, Soutto M, Washington MK, Revetta F, Ecsedy J, Zaika A, Rau TT, Schneider-Stock R, Belkhiri A, El-Rifai W. The aurora kinase A inhibitor MLN8237 enhances cisplatin-induced cell death in esophageal adenocarcinoma cells. Mol Cancer Ther. 2012; 11: 763-774.

40. Liu Y, Hawkins OE, Su Y, Vilgelm AE, Sobolik T, Thu YM, Kantrow S, Splittgerber RC, Short S, Amiri KI, Ecsedy JA, Sosman JA, Kelley MC, Richmond A. Targeting aurora kinases limits tumour growth through DNA damagemediated senescence and blockade of NF- $\mathrm{B}$ impairs this drug-induced senescence. EMBO Mol Med. 2013; 5: 149166.

41. Mohan P, Castellsague J, Jiang J, Allen K, Chen $\mathrm{H}$, Nemirovsky O, Spyra M, Hu K, Kluwe L, Pujana MA, Villanueva A, Mautner VF, Keats JJ, et al. Genomic imbalance of HMMR/RHAMM regulates the sensitivity and response of malignant peripheral nerve sheath tumour cells to aurora kinase inhibition. Oncotarget. 2013; 4: 80-93. 